\title{
Editorials
}

\section{Assessing, communicating, and managing risk in general practice}

\section{WHAT IS RISK ASSESSMENT, COMMUNICATION, AND MANAGEMENT?}

Risk assessment, communication and management has become a topic of increasing interest across many sectors of society: from stockbrokers to firefighters to politicians. One of the most complex areas of its application is 'health', with general practice arguably being one of the most complex contexts within that sector.' The Oxford Dictionary defines 'risk' more broadly as 'a situation involving exposure to danger' and current clinical definitions often define 'risk communication' as 'the open two-way exchange of information and opinion about risk, leading to better understanding and better decisions about clinical management'. ' 'Risk management' on the other hand, involves the forecasting and evaluation of risks together with the identification of procedures to avoid or minimise their impact'. Better 'risk communication' is therefore one potential strategy for 'risk management'. ${ }^{3}$

\section{RISK ASSESSMENT AND THE COMPLEXITY OF GENERAL PRACTICE}

This issue of the BJGP focuses on Risk \& Safety with several papers highlighting the complexity of risk assessment, communication and management in general practice. Spencer and colleagues ${ }^{3}$ take a 'systems-level' approach to risk management through refinement of a set of prescribing safety indicators by a panel of GPs and a subset considered to be associated with high or extreme risk to patients. The authors plan to develop a computer-based toolkit enabling individual GP audits, citing the PINCER trial as a successful intervention for reducing prescribing errors. ${ }^{4}$ However, the PINCER intervention focused on only three prescribing indicators and was a complex intervention involving pharmacistled educational outreach, computerised feedback, localised solution development and ongoing support compared with feedback alone. Spencer and colleagues' study highlights the challenge of scaling up risk management strategies for GPS, as they have identified 23 high priority indicators within a total list of $56 .{ }^{3} \mathrm{GPs}$ face a great diversity of prescribing decisions and patients with multiple comorbidities throughout each working day. Indeed, lessons from the PINCER trial suggest

"There is also a perceived tension between

population-level quality frameworks and the

underpinning principles of general practice

none of which are easily captured or identifiable

in most quality frameworks

that processes such as better interprofessional communication, support, and locally-tailored solutions warrant closer scrutiny and may also go some way towards addressing high-risk use of overthe-counter medication as highlighted by Koffeman and colleagues. ${ }^{5}$ It is perhaps the lack of attention to these 'less easilymeasured' process factors and the need for greater flexibility in systems-level risk management that have brought much of the criticism of initiatives such as the UK's Quality and Outcomes Framework. ${ }^{5}$

\section{HOW DOES RISK COMMUNICATION ALIGN WITH THE CORE CHARACTERISTICS OF GENERAL PRACTICE?}

There is also a perceived tension between population-level quality frameworks and the underpinning principles of general practice. General practice is characterised by patient-centredness, the pivotal role of the doctor-patient relationship, advocacy, the biopsychosocial model, multidisciplinarity, multimorbidity and continuity of care, none of which are easily captured or identifiable in most quality frameworks. These core characteristics reflect an interactive and personalised process at the individual patient-level. It's appropriate then, that two articles in this issue ${ }^{6,7}$ also focus on risk communication and stratified approaches to risk factor assessment and management. They illustrate how risk communication can be linked to quality and safety in general practice defined as the best health outcomes possible, given the available circumstances and resources consistent with patient-centred care'.

In this issue, Black and colleagues have modelled cardiovascular outcomes in patients with diabetes over 10 years, having divided their cohort at baseline into four absolute CVD risk strata using their UKPDS score. ${ }^{7}$ The group with the highest risk at baseline had the greatest reduction in CVD risk with treatment regardless of socioeconomic status. They suggest that this kind of approach to risk assessment allows for more individualised patient goal setting and risk factor management, and it is very encouraging to see the potential to reduce health inequalities. Similar riskstratification has been proposed for cancer screening and is not only synergistic with the core principles of general practice but also the new era of personalised medicine. ${ }^{8}$ A number of risk assessment models and tools for CVD and cancer have become available to GPs and their patients in recent years and debate continues as to which ones are best.? Regardless of the risk assessment tool chosen, it is the way

\section{"While there should be efforts to train practitioners in risk communication, there is a need to ensure that they are provided with good quality tools to use in clinical practice, particularly in the complex environment of general practice...}


the risk assessment is used in clinical decisions which really counts. Black and colleagues' work shows the benefit of intensively treating diabetic patients with higher CVD risk with less impact on CVD risk if the absolute risk at baseline is low, validating the risk-stratified approach to management. ${ }^{10}$ However, one of the features of general practice is that most patients will be of low or moderate risk and the recent changes in risk thresholds for statin use highlight the ongoing uncertainty about how to apply risk models and trial data in 'real world' clinical practice. ${ }^{11}$ GPs do, however, seem to use absolute CVD risk assessment tools in a variety of ways ranging from dismissing them, to using them as education and behaviour change tools to encourage behaviour change. ${ }^{12}$

It is the two-way exchange defined by 'risk communication' that perhaps holds the key to better risk management in the general practice context. Some of the most promising evidence of effect has been in trials of patient decision aids, particularly where they include both practitioners and patients. ${ }^{13}$ Strategies targeting only GPs have had limited effect.10 This aligns well with the principles of patient-centred care and the value of the doctor-patient relationship.

\section{APPLYING NEW EVIDENCE ON 'HOW' TO COMMUNICATE CAN HELP GPS AND THEIR PATIENTS}

However, as Harmsen and colleagues point out, ${ }^{6}$ the way that information is presented can affect perceptions of risk and GPs need to be mindful of this as they engage their patients in risk management discussions. There are now 115 randomised controlled trials in the Cochrane review of patient decision aids and a strong evidence-base that presenting outcomes quantitatively improves the accuracy of risk perception. ${ }^{14}$ Furthermore, decision aids incorporating evidence-based standards result in better quality decisions. ${ }^{15}$ While there should be efforts to train practitioners in risk

\section{ADDRESS FOR CORRESPONDENCE}

\section{Lyndal Trevena}

Room 321b, Edward Ford Building (A27), University of Sydney NSW 2006, Australia.

\section{E-mail: lyndal.trevenaßssydney.edu.au}

communication, there is a need to ensure that they are provided with good quality tools to use in clinical practice, particularly in the complex environment of general practice where such a diverse range of clinical problems are addressed each day. General principles of good risk communication are increasingly well-defined. They include using numeric estimates to improve accuracy of risk perception, choosing a format that matches the cognitive task, defining a relevant denominator for your audience, using a consistent format, considering the target groups graph literacy and numeracy when selecting formats and the magnitude of estimates as format bias may exist particularly with very small numbers. ${ }^{16}$

In summary, risk assessment, communication, and management in general practice needs to take into consideration the complexity of the clinical environment and the underpinning characteristics and principles that sets it apart. Systems-level approaches appear to have potential but their ability to be scaled up is likely to require flexibility for local adaptation. There is promising evidence for the potential impact of risk communication to individualise goal setting with patients, improve adherence and reduce inequalities. This appears to have the greatest effect when both practitioners and their patients are engaged in the process.

\section{Lyndal Trevena,}

Associate Professor, University of Sydney, Sydney.

\section{Provenance}

Commissioned; not externally peer reviewed.

DOI: 10.3399/bjgp14X677725

\section{REFERENCES}

1. Sturmberg JP, Martin CM, Katerndahl DA. Systems and complexity thinking in the general practice literature: an integrative, historical narrative review. Ann Fam Med 2014; 12(1): 66-74

2. Edwards A, Elwyn G, Mulley A. Explaining risks: turning numerical data into meaningful pictures. BMJ 2002; 324(7341): 827-830.

3. Spencer R, Bell B, Avery AJ, et al. Identification of an updated set of prescribing-safety indicators for GPs. Br J Gen Pract 2014: DOI: 10.3399/bjgp14X677806.

4. Avery AJ, Rodgers S, Cantrill JA, et al. A pharmacist-led information technology intervention for medication errors (PINCER): a multicentre, cluster randomised, controlled trial and cost-effectiveness analysis. Lancet 2012; 379(9823): 136-142.

5. Koffeman AR, Valkhoff VE, Celik S, et al. Highrisk use of over-the-counter non-steroidal anti-inflammatory drugs: a population based cross-sectional study. Br J Gen Pract 2014; DOI: 10.3399/bjgp14X677815.

6. Harmsen CG, Kristiansen IS, Larsen PV, et al. Communicating risk using absolute risk reduction or prolongation of life formats: cluster-randomised trial in general practice. $\mathrm{Br}$ $J$ Gen Pract 2014; DOI: 10.3399/bjgp14X677824.

7. Black JA, Sharp SJ, Wareham NJ, et al. Change in cardiovascular risk factors following early diagnosis of type 2 diabetes: a cohort analysis of a cluster-randomised trial. Br J Gen Pract 2014 DOI: 10.3399/bjgp14X677833.

8. Burton $\mathrm{H}$, Chowdhury S, Dent T et al Public health implications from COGS and potential for risk stratification and screening. Nat Gen 2013; 45(4): 349-351.

9. Tunstall-Pedoe H. Cardiovascular risk and risk scores: ASSIGN, Framingham, QRISK and others: how to choose. Heart 2011; 97(6): 442-444.

10. Vagholkar S, Zwar N, Jayasinghe UW, et al. Influence of cardiovascular absolute risk assessment on prescribing of antihypertensive and lipid-lowering medications: a cluster randomized controlled trial. Am Heart J 2014; 167(1): 28-35.

11. Anonymous. Statins for millions more? Lancet 2014; 383(9918): 669.

12. Bonner C, Jansen J, McKinn S, et al. General practitioners' use of different cardiovascular risk assessment strategies: a qualitative study. Med J Aust 2013; 199(7): 485-489.

13. Krones T, Keller H, Sönnichsen A, et al. Absolute cardiovascular disease risk and shared decision making in primary care: a randomized controlled trial. Ann Fam Med 2008; 6(3): 218-227.

14. Stacey D, Legare F, Col NF, et al. Decision aids for people facing health treatment or screening decisions. Cochrane Database Syst Rev 2014; 1: CD001431.

15. Gentles SJ, Stacey D, Bennett C, et al. Factors explaining the heterogeneity of effects of patient decision aids on knowledge of outcome probabilities: a systematic review sub-analysis. Syst Rev 2013; 2: 95.

16. Trevena L, Zikmund-Fisher B, Edwards A, et al. Presenting quantitative information about decision outcomes: a risk communication primer for patient decision aid developers. BMC Med Inform Decis Mak 2013; 13(Suppl 2): S7. 